\title{
Multiple Layer Scanning in Magnetopneumography
}

\author{
Jiří Tomek, Antonin Platil, and Pavel Ripka, Member, IEEE
}

\begin{abstract}
In magnetopneumography (MPG), we scan weak remanent magnetic fields (in the range of $n T$ ) of ferromagnetic dust deposited in the lungs of affected workers. This is carried out in an unshielded lab. Variations of the background field during the scanning period bias the inversion process, i.e., the estimation of the magnetic sources from the measured field. We present an innovative mathematical technique of finding a field equation uniquely describing the magnetic moments in the nodes of the measurement space. Simultaneously, it enables the tracking of the values of the disturbing gradient created by external distant sources during the time of scanning. Our method preserves the first-order gradients obtained in multiple layers (scan heights above the object) instead of losing data with the higher order gradients which are not effective here. Six coaxially aligned fluxgates were used for scanning the field maps. The obtained five first-order gradients for each node give us the parameters of the respective "equivalent" magnetic sources. Furthermore, we obtain a parameter describing the error originating mainly from the background field. The main limits of the presented method are: 1) the alignment of the probes; 2) their number being limited by a minimum distance; and 3) nonhomogeneity of the external field.
\end{abstract}

Index Terms-Computational elimination of disturbances, magnetopneumography, multiple layer scanning.

\section{INTRODUCTION}

$\mathbf{M}$ AGNETOPNEUMOGRAPHY (MPG) was proposed in 1970s [1] as a noninvasive diagnostic method for repetitive screening of lungs of workers exposed to aerosols containing ferromagnetic particles. The subjects (or phantoms) are first magnetized in a strong DC field. Subsequently, we can measure the weak magnetic field of the deposited particles by a suitable gradiometric configuration of the probes-for this purpose, we use fluxgates [2]-[5]. For MPG (as well as magnetoencephalography [MEG]) far more sensitive, but also far more expensive and cryogenic temperatures demanding Superconducting Quantum Interference Devices (SQUIDs) are typically used (e.g., [6] and [7]). The applicability of the

Manuscript received January 03, 2009; accepted January 09, 2009. Current version published February 27, 2009. This work was supported by the Research Program MSM6840770012 "Transdisciplinary Research in the Area of Biomedical Engineering II" at CTU in Prague, sponsored by the Ministry of Education, Youth and Sports of the Czech Republic. This is an expanded paper from the Sensors 2007 Conference and was published in its proceedings. The associate editor coordinating the review of this paper and approving it for publication was Dr. Subhas Mukhopadhyay.

J. Tomek is with the Faculty of Electrical Engineering, Department of Measurement, Czech Technical University, Prague, Czech Republic, and also with the Medical Technique Service Department, General Teaching Hospital Prague, Prague, Czech Republic (e-mail: tomekj1@fel.cvut.cz).

A. Platil and P. Ripka are with the the Faculty of Electrical Engineering, Department of Measurement, Czech Technical University, Prague, Czech Republic (e-mail: platil@fel.cvut.cz; ripka@fel.cvut.cz).

Color versions of one or more of the figures in this paper are available online at http://ieeexplore.ieee.org.

Digital Object Identifier 10.1109/JSEN.2009.2014409 fluxgate gradiometers for MPG has been demonstrated in [2]. We have shown that the amounts of magnetic deposits in lungs of metal workers may reach several grams [2]-[4] with a remanent field of several hundreds $\mathrm{nT}$ in the distances we use. These fields can be clearly detected by fluxgates.

The measured magnetic field data can be used to determine the dust load in lungs [2]-[12] and also eventually its distribution, for which we have used artificial neural networks with partial success [10], [11]. However, the field variations during the step-by-step scans (several minutes for all the nodes) bias the field-maps considerably. These make the inversion difficult [4], [9], [10]. Furthermore, the difference between the signals (the "gradient") does not uniquely describe the measured field and additionally there is the nonuniqueness of the inversion of the general magneto-static problem [13]-[17]. Our aim is to develop a portable system that does not need any shielding and no cryogenic equipment, like the SQUID magnetometers, e.g., in MEG. MEG even in the shielding also usually employs higher order gradients to better suppress the background noise [17]-[21]. On the other hand, fluxgates have much higher noise than SQUIDs. Low-temperature SQUID sensors have noise in units of fT Hz ${ }^{-1 / 2} @ 1 \mathrm{~Hz}$ (e.g., [16] and [22]). In contrast, our device has a noise of $13 \mathrm{pTHz}^{-1 / 2} @ 1 \mathrm{~Hz}$ [4]. Slightly better parameters of fluxgate gradiometer are reported in [23].

MEG systems use large and dense arrays of SQUID sensors (typically hundreds). These are usually triggered simultaneously (e.g., [18]-[20]). In our application, however, we cannot place the fluxgate sensors so close to each other because of the feedback field created by the compensating coils and because of the ferromagnetic cores of the sensors, it would result in strong interference. Here, we work with a $10 \mathrm{~cm}$ gradiometric base (an axial distance between the sensors), for which the influence between sensors is less than $1 \%$. MEG SQUID arrays have the pickup coils several millimeters apart and even the gradiometric bases can be smaller, e.g., less than $70 \mathrm{~mm}$ [22]. This distance between the sensors naturally depends on the application and balance between the sensitivity and noise suppression, thus the length is a calculated optimum for a particular application [16], [24].

Our system has to employ a sequential mode of measurement to cover all the scanning areas in the proper resolution. This mode is more susceptible to a background field gradient variation, which can be tens of $\mathrm{nT}$ in a short time between the measurement points. Some authors use methods of tracking the background by a separate reference gradiometer [16], [25] in order to subtract it from the useful signal, but our study with fluxgates has shown that this approach has serious limitations in the real environment of an ordinary laboratory [11]. This finally motivated us to experiment with solutions that enable a combination of our two basic needs: a) evaluating more data acquired in several layers above the tested object and b) the possibility to determine and suppress the background field variations. The 


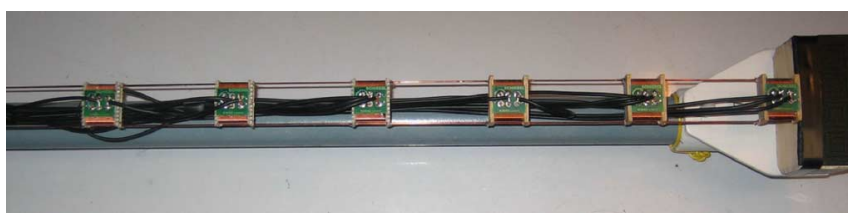

Fig. 1. Design of the six-probe gradiometer. Probes are aligned coaxially on a pair of tight copper wires $10 \mathrm{~cm}$ from each other.

setup presented below enables a number of gradiometric experiments, while having all the sensors fixed vertically above one point in space. In this paper, we present an idea of processing the data from several coaxial first-order gradiometers (FoG) using this fluxgate system.

We have used single "FoGs" in all previous research [2]-[5], [10], [11], [26], [27]. The former setup allowed satisfactory estimates of the total dust load [12] and made the determination of the simple compact artificial sources possible [5], [10], [11]. Using such a setup, the time variations of the background gradient during scanning could not be recorded and suppressed. This limitation makes the in vivo tests during the daytime impossible (mainly due to disturbances from electric traction and other traffic). Note that the field variations often reach $200 \mathrm{nT}$ p-p, and the FoG variations more than $20 \mathrm{nT} / \mathrm{m}$ p-p [3], [4]. Therefore, a logical step was adding more sensors to the system. As mentioned above, these can also be utilized to obtain higher order gradients. This approach is used in MEG [16]-[21]. Alternatively, it is possible to obtain more data results in one scan and even to subtract the variations of the background field by fitting the data into an experimental formula. Such a fitting needs a proper equation derived from the Biot-Savart Law and the utilization of correct initial conditions derived from the models. This approach in contrast, is capable of providing us with clearer datasets for a more successful inversion. It may also help to decrease the sensitivity to measurement errors and to the nonhomogeneity of the background field and its changes. The revealed technique would theoretically require as many coaxial probes as possible. However, as discussed above, we are limited by the mutual influence of the sensors, which makes denser spacing almost impossible.

The tests of the artificial disturbing sources, as presented, e.g., in [20], are to be subject of further experiments. In this paper, we use the data measured in a lab influenced by distant magnetic fields from the operation of subway, trams, and other city traffic [4], [12], but without strong interference from any close fields.

\section{The Measurement EQuiPMent}

The basic procedures of our MPG measurements were already described, e.g., in [10] and [11]. Subjects or phantoms that are to be examined must be magnetized in a strong DC field prior to scanning in order to have properly aligned magnetizations. For the measurements, a static gradiometer composed of several fluxgate probes is used-see Figs. 1 and 2. All sensors are aligned in a row, all having their sensitivity axis in the same direction (vertically when over the bed), with minimal angular deflections (less than $1^{\circ}$ ) from the optimum. The particular gradients are calculated by the software from six separate channel readings. The parameters of the sensors, electronics, and the gra-

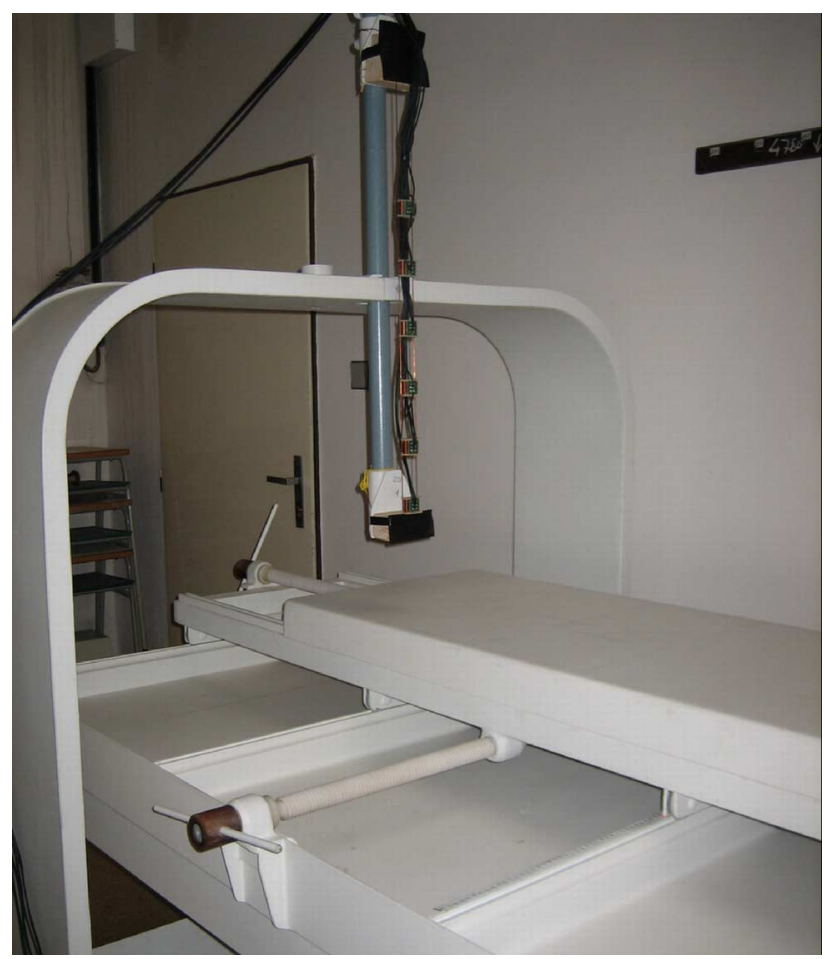

Fig. 2. Measuring magnetopneumographic bed with the gradiometer fixed into a separate portal.

diometric configuration were fully described in [4] and the utilization of all six channels in [26], [27]. The key parameters are: sensitivity $133 \mathrm{mV} / \mu \mathrm{T}$ and range $+/-130 \mu \mathrm{T}$. The bandwidth is limited by the low-pass filter $(20 \mathrm{~Hz})$ rather than by the excitation frequency of $8 \mathrm{kHz}$.

The gradiometer's probes are, in our application, just above the bed, nevertheless, quite usually some sensors are even placed below the subject [9]. The gradiometer is fixed to a portal that is separate from the movable scanning bed in order to isolate any mechanical vibrations, see Fig. 2.

We need to move the measured object, not the gradiometer, which has to be stationary. This is necessary because even a minor position change of the gradiometer in the Earth's field results in a great change in the detected signal, higher than the signal we want to detect. That is why it is necessary to keep the probes steady.

Formerly, we used just one first-order gradiometer made of two coaxially fixed probes at a certain distance, however, data from a single plane scan over the tested subject/phantom is not sufficient for determining the distribution of the particles in the lungs. Furthermore, the variations of the background field, which cannot be detected by this configuration, degrade the data. Later on, we have made experiments with three sensors and finally with six sensors.

\section{EXPERIMENTAL SIX-PROBE GRADIOMETER}

\section{A. Why Six Probes?}

The main reasons are to get more scanning heights, thus more field maps can be scanned during one run, and to make experiments with suppression of background field changes, that are 
not negligible during the period of scanning. The scanning period takes, for the minimum number of nodes (144) in the plane, approximately $20 \mathrm{~min}$ and the peak to peak signal change due to the background fields variations can be up to $20 \mathrm{nT} / \mathrm{m}$ in our lab.

The fifth and sixth (furthest) sensors are not too influenced by the measured nearby field. Therefore, we can use the topmost two sensors as a compensating first-order gradiometer and we could obtain four second-order gradients when subtracting the measured fields from the individual probes or three third-order gradients, i.e., (first-second) - (fifth-sixth) and (second-third) - (fifth-sixth ), etc. There are also other possibilities how to process the signals, but we will stick with the idea mentioned in the Section I utilizing five first-order gradients and a mathematical evaluation of the background gradient.

\section{B. Building the Gradiometer}

We have used a rigid glass-fiber reinforced composite pipe and fixed to it two firm, totally nonmagnetic holders on which two copper wires of $1.4 \mathrm{~mm}$ diameter have been tightly stretched. Before that, small holes were drilled through the sensors' frames and these were slipped over and mounted onto the wires. The stretching of the wire was realized by a set of wooden wedges. To ensure that the weight of the signal cables will not change the probes' positions these were fixed to the top holder. The fitted tube can be fixed easily to the portal above the magnetopneumographic bed (see Fig. 2) and moved upwards or downwards to set the desired distance from the measured object.

\section{Real Gradiometer Properties}

Although the mounting was made very precisely, the gradiometer is not well balanced. The reason for this is that the sensing direction of each sensor differs from its geometrical axis. This is usually caused by nonhomogeneity of the core or winding, or deviations in the core position. Another problem is that in order to achieve better linearity, a wider range and better long-term stability of the sensitivity, each channel of our magnetometer uses individual negative feedback. The pickup coil of each fluxgate is also used to create a DC compensation field. It means that the field of the compensating coils may influence the other probes. This was very clear during calibration of the channels in the Helmholtz coils. The conversion constants (sensitivities) were approximately $1 \%$ lower when the neighboring sensors were also connected and creating the compensation fields inside them. Even the sensors $20 \mathrm{~cm}$ apart had a certain mutual influence.

\section{Software for the Measurements and the Modeling}

The output voltages from the separate magnetometer's channels, proportional to the measured magnetic field values, were synchronously (using a trigger function) digitalized by six Agilent 34401 multimeters and transferred to a host PC via an IEEE 488 bus using a Matlab Instrument Control Toolbox and custom-made script. To ensure that just the wanted gradients are measured, the first scan (just one extra node) has been made without the object in order to have the initial record of the

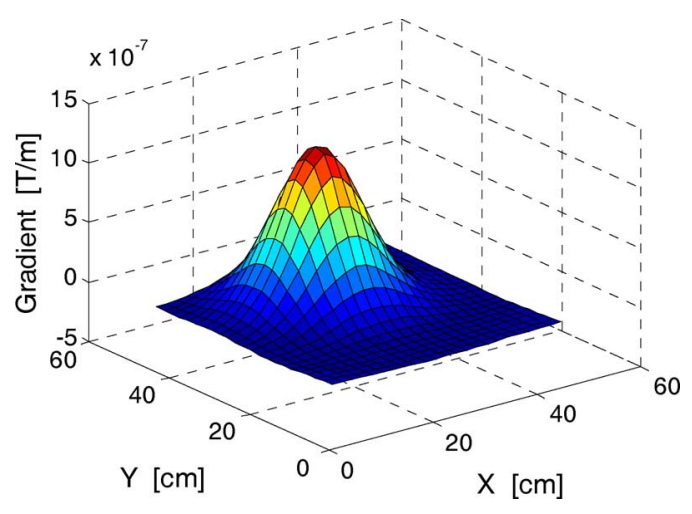

Fig. 3. Modeled gradient ${ }_{1}$, first-second sensor.

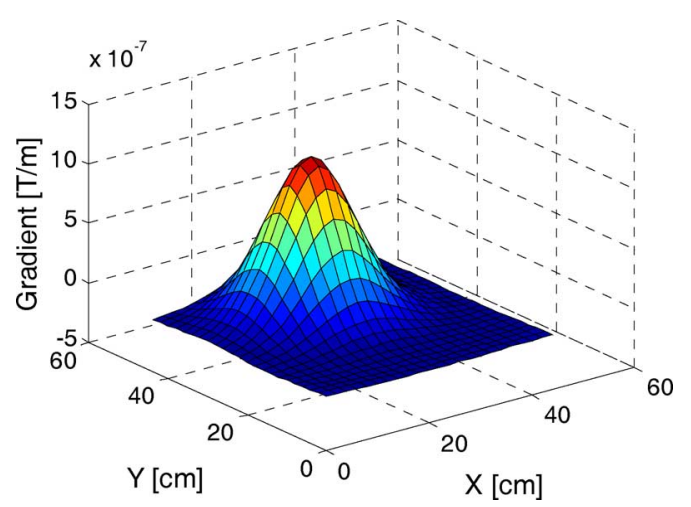

Fig. 4. Measured gradient ${ }_{1}$, the first sensor is $18 \mathrm{~cm}$ from the bed.

gradients caused by the background field and only after that the examined object was placed on the movable bed and scanned. The first dataset has been always subtracted from every scan.

The computer forward model already described in [5], [10], and [11] has been modified to process more scanning heights.

\section{MeAsurements}

The experiments were conducted just with the phantom described in [10] and [11]. It is composed of 574 cubes $(2 \times 2 \times$ $2 \mathrm{~cm}^{3}$ each) and has a total volume of $4592 \mathrm{~cm}^{3}$. The measured compact sources were composed of several adjacent phantom cubes containing ferromagnetic material. Here, we present one measurement with a source composed of 16 magnetized phantom cubes ( 2 in width $-X, 4$ in length $-Y$, and 2 in height $-\mathrm{Z}$ ). Each of these plastic cubes contains $80 \mathrm{mg}$ of magnetite $\left(\mathrm{Fe}_{3} \mathrm{O}_{4}\right)$ powder homogenously spread in the volume. After magnetization, Ampere's magnetic moment $\mathrm{m}_{\mathrm{A}}$ of such a cube was around $300 \times 10^{-6} \mathrm{Am}^{2}[5]$.

In the figures above, there are field-maps depicted in two heights, i.e., scanning planes above a model or a phantom (measured object). The first set (Figs. 3 and 4) represents the gradient between the first and second sensor-designated "gradient ${ }_{1} . "$ The second set (Figs. 5 and 6) represents the gradient between the fourth and fifth sensor-designated "gradient 4 "-and it is rather noisy, mainly due to the background field variations. The measured gradients over a magnetized phantom (Figs. 4 and 6) correspond well with the models in Figs. 3 and 5, respectively. 


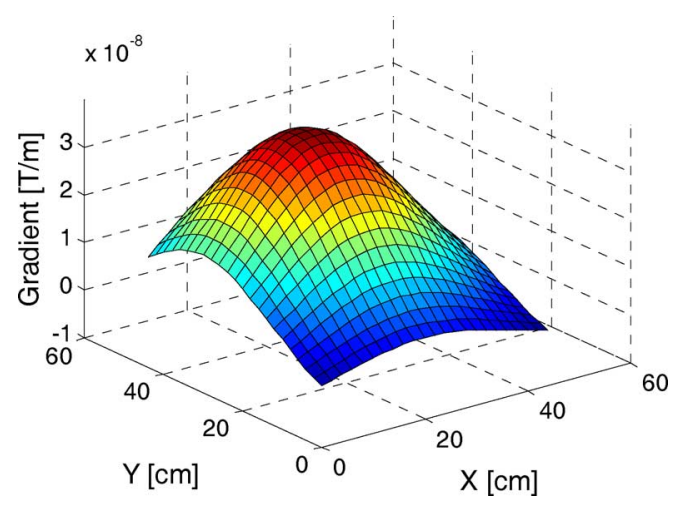

Fig. 5. Modeled gradient ${ }_{4}$, fourth-fifth sensor.

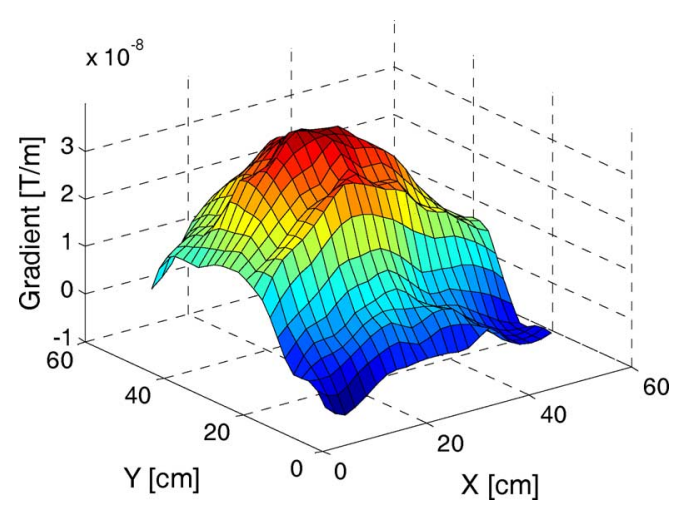

Fig. 6. Measured gradient ${ }_{4}$, the fourth sensor is $48 \mathrm{~cm}$ from the bed.

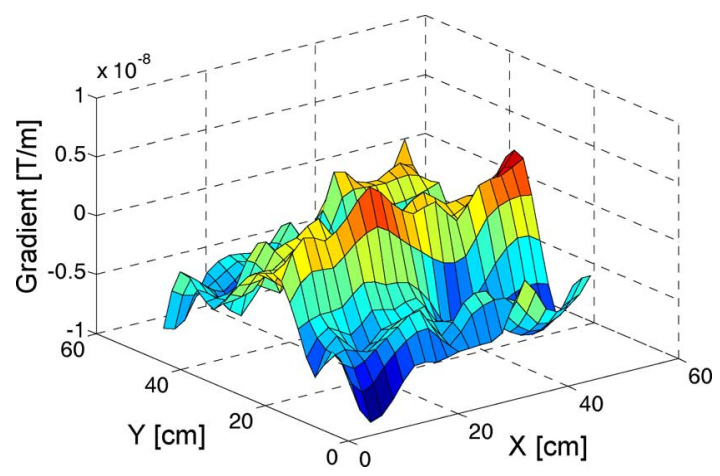

Fig. 7. Scan of the background gradient during an experiment without any source (object); actually it is "gradient ${ }_{4}$ " with the fourth sensor $48 \mathrm{~cm}$ from the bed.

One can see well that the noise in the graphs of the real measurements is not negligible.

The most distant gradient (between the fifth and sixth sensor) is buried in the noise, thus incomparable with the computer model. To imagine the gradient variations during scanning, we present a background gradient map, i.e., the measurement without the source. In Fig. 7, there is the noise in "gradient 4 " between the points at the heights 48 and $58 \mathrm{~cm}$ above the bed. For every scan, this noise map is different; these are, in fact, the " $D$ terms" (see Section V) in each node.

The field variations, especially during the day time in urban areas, are significant. One can also see that the measured gradients have certain offsets in contrast to computer models, while

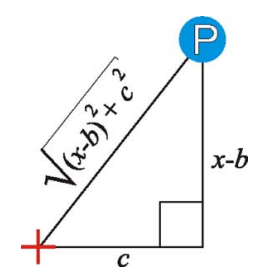

Fig. 8. The meaning of the variables in (2). The $P$ in the circle stands for the closest probe of the gradiometer and the cross symbolizes the center of the equivalent magnetic dipole of the measured source.

the shape is almost identical. Also, this should be suppressed by the proposed mathematical method.

\section{FITTING EXPERIMENTS}

The described method below is primarily used to eliminate the field variations in scans and furthermore, to condense data for further processing. The mathematical background is given by two equations suited to our problem, see (1) and (2). Equation (1) takes signals from two neighboring sensors and calculates the "gradient" by simple subtraction, the remaining constant $D$ is the additional gradient due to the background sources, which we try to eliminate

$$
\text { Gradient }=P_{X 1}-P_{X 2}+D .
$$

Equation (2) is derived from the Biot-Savart Law [28]. It calculates the magnetic field of an ideal dipole magnetized in the direction of our gradiometer's axis

$P_{X \mathrm{i}}=A \cdot\left\{\frac{3 \cdot(x-b)^{2}}{\left[(x-b)^{2}+c^{2}\right]^{5 / 2}}-\frac{1}{\left[(x-b)^{2}+c^{2}\right]^{3 / 2}}\right\}-K$

where

$A$ is a parameter combining the magnetic moment of the source and proportionality constants;

$b \quad$ is the height of the center of the source above the zero level, which is the surface of the scanning bed;

$c$ is the perpendicular (horizontal) distance of the center of the calculated dipole from the scanning node;

$K$ is the additive constant, in real data it stands for the background field variation, in the models it could be omitted.

The meanings of $c$ and $b$ from (2) are also explained in Fig. 8.

The $P_{X \mathrm{i}}$ from (2) for a particular height (i=1 to 6) without the constant $K$ comes to (1) that calculates the gradient between the respective sensors. If the sensors are exactly in a line and if the field in the region of the fluxgates caused by far sources has a constant gradient, it should be possible to determine the added value as an offset $D$ in (1). In the gradient function (1), the additive constants $K$ from (2) for different probes are partly subtracted and the described term $D$ remains.

The revealed method has been implemented by a custommade script in Matlab based on Nelder-Mead optimization algorithm with the least squares norm. The quality of the fitting 


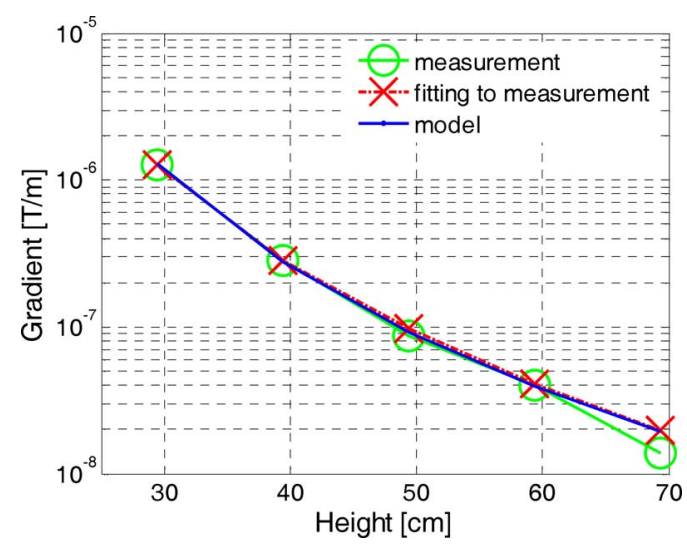

Fig. 9. Comparison of the modeled gradient to the measured data and the fitting to this data according to the parameters derived from the model. The measurement has been taken at the coordinates $\{20,28\}$ (scan by $4 \mathrm{~cm}$ steps) and the center of the source was at $\{20,30\}$.

depends on the well-set initial conditions and the amount of data to be fitted into the described expression for the measured gradients.

Considering the height of the lung phantom being $18 \mathrm{~cm}$ and 5 more centimeters as a distance for other tissue and clearance for breathing, the reasonable distance of the closest probe's edge is $23 \mathrm{~cm}$ above the moving bed. The fittings for any node above the phantom containing some magnetic sources (even with a random distribution of the contaminated model cubes in it) worked well.

Let us start with the simple case that was demonstrated in Section IV. Here, the closest probe is $18 \mathrm{~cm}$ above the bed. We have the compact homogenous deposit simulated by 16 adjacent cubes on the bed's surface.

As was already mentioned we lose the information about the absolute values when using gradients and determining of the source is, due to this, nonunique [13]-[17]. The main benefit of the presented method is the number of the correlated first-order gradients. These enable a mathematical determination of the interesting information and the exclusion of some unwanted signals. With this approach, we can obtain clearer data for further processing. The signals to be eliminated are the far field variations between the probes (term $D$ ). The process can determine them and then they can be subtracted for each scanning node separately. In our case, the fitting also acted as a compensation for the inaccurate measuring setup, see Figs. 9 and 10.

The optimization applied on the measured data gave some very good results that are presented in Table I and in Figs. 9 and 10. The label "measurement" designates the gradients measured over a phantom source; "model" gives the calculated interpolated gradient curves for the same source. Finally, the fitted results "fitting to measurement" that are drawn as dash-dotted curves are the real measured data ("measurement") after processing by the described method. Fig. 9 depicts the measurement close to the maximum of the measured field (almost above the compact source) and has the character of $1 / \mathrm{x}^{3}$ and the fitting works perfectly because of strong signals compared to the noise.

On the contrary, in the second measurement (Fig. 10) taken close to the edge of the measurement plane (far from the center

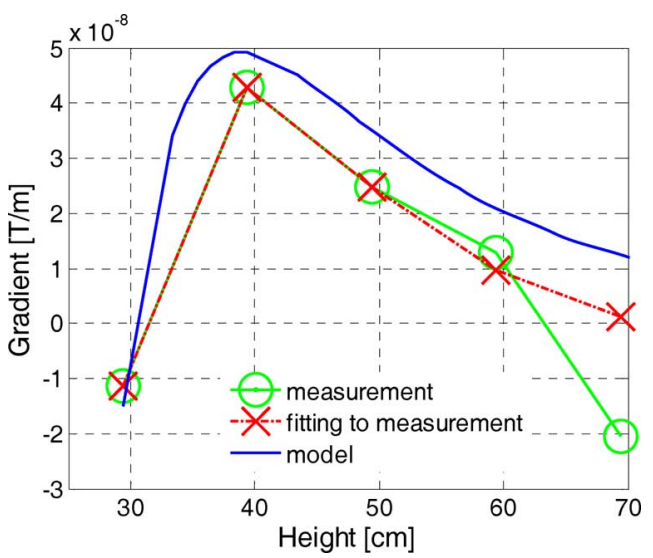

Fig. 10. Comparison of the gradients far from the maximum-coordinates $\{8,18\}$, model and measurement. Background noise causes bigger differences with the increasing height above the bed.

TABLE I

PARAMETERS DERIVED USING THE OptimizATION PROCESS From the Measured Data. The Compact Source $(4 \times 8 \times 4 \mathrm{~cm})$ HAD the CENTER AT THE COORDinAtes $\{x=20, y=30\}$.

\begin{tabular}{|c|c|c|}
\hline Parameters & $\begin{array}{c}\text { Measurement at } \\
\text { coordinates }\{20,28\}: \\
\text { result / ideal result }\end{array}$ & $\begin{array}{c}\text { Measurement at } \\
\text { coordinates }\{8,18\}, \text { low } \\
\text { field from the source: } \\
\text { result / ideal result }\end{array}$ \\
\hline$A(-)$ & $0.0048 / 0.0048$ & $0.0046 / 0.0048$ \\
\hline$b(\mathrm{~cm})$ & $2.0190 / 2.0000$ & $1.7822 / 2.0000$ \\
\hline$c(\mathrm{~cm})$ & $2.0604 / 2.0000$ & $17.6627 / 16.9706$ \\
\hline$D(T / \mathrm{m})$ & $-5.8817 \times 10^{-10}$ & $-1.1053 \times 10^{-8}$ \\
\hline
\end{tabular}

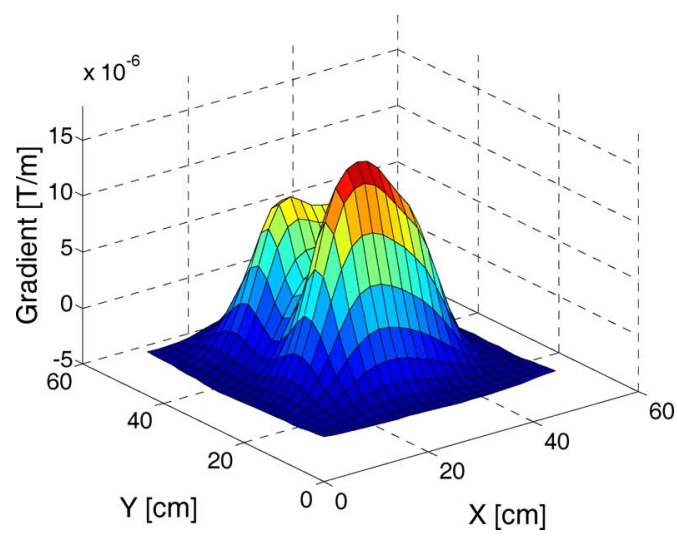

Fig. 11. Modeled first gradient for a randomly generated source containing 277 magnetite-contaminated cubes out of the total 574 cubes composing our phantom of the lungs.

of the compact source), the fitting is more influenced by the imperfections in fixing the probes to the tube and by noise, see comparisons in Table I.

More complex sources were simulated by random distribution of magnetite containing cubes in the whole volume of the lung phantom. We were modeling it with the closest gradiometer probe $23 \mathrm{~cm}$ above the bed. In the presented case, the number of magnetic cubes was 277 out of 574, each having Ampere's magnetic moment $300 \times 10^{-6} \mathrm{Am}^{2}$. The first gradient map (Fig. 11) shows two lungs clearly. 


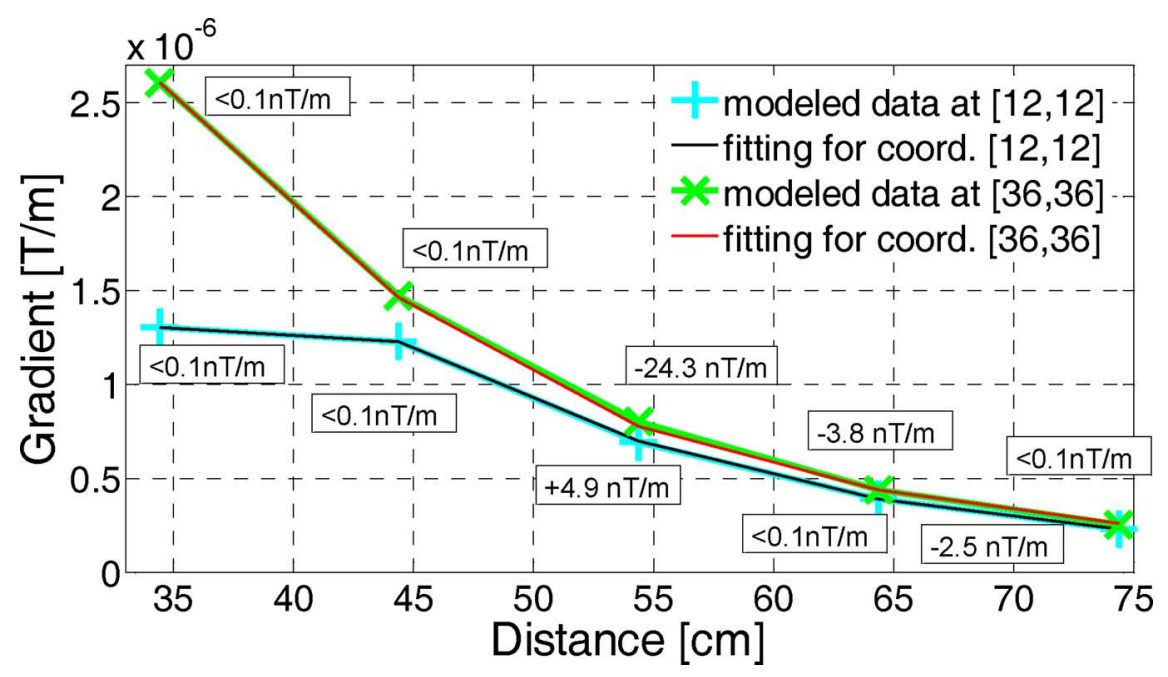

Fig. 12. Comparison of modeled and fitted gradients at two different coordinates of the measuring plane. It shows eligibility of the formula for fitting. The axis of values (distance) starts at the height of the second probe of the gradiometer. The numerical insets show fitting errors in individual distances.

Nevertheless, we can still fit all five correlated gradients to the mentioned formula. The fitting results differ for each of the scanning nodes, so we get estimates of many "equivalent" dipoles characterizing the measured field. This condensed information can later be used as an input of a neural network for an inversion process determining the dust load distribution. Also, in this case, the data enables the evaluation of the background field bias, though just five gradients are the minimum required for this kind of mathematical processing. The reason is the possibility of a bad setting of the initial conditions for the optimization process as the chosen Nelder-Mead algorithm may stop in some local minimum, resulting in an erroneous fit. Thus, we have finally used a random setting of the algorithm initial conditions and by an iterative procedure of their refining, the best possible result has been achieved. It is necessary to consider that we are fitting a continuous equation into the data coming from the discrete model, which may also cause a problem with convergence. The fitting to the modeled data is sometimes imperfect and differences of even several $\mathrm{nT} / \mathrm{m}$ appear (look at the differences shown in the insets of Fig. 12). We are presenting three sample fittings to the (simulated) gradients taken on the diagonal of the scanning area below, i.e., at three positions across the lungs (Table II). Two of them are also in Fig. 12. As the fitted data comes from the computer model, i.e., there is no bias from the additional background gradient, the $D$ terms are extremely low. The results demonstrate (as may be expected) that the $A, b$, and $c$ parameters differ for each scanning node above a complex source. The individual data results are useless, only the whole set can be used for further processing. The field in the node of the coordinates $\{36,36\}$ has even such a character that the center of an equivalent dipole that can create it, is below the scanning bed. However, the importance is in the possibility to be able to describe the complex measurement in respective nodes with only four parameters (de facto three, as $D$ stands for errors and variations) and use them for inverse processing, which is our primary motivation.

From the distance of the fourth gradient, which was $53 \mathrm{~cm}$ above the bed ( $35 \mathrm{~cm}$ above the phantom), the field starts to have
TABLE II

PARAMETERS Derived USING THE OPTIMIZATION PROCESS From THE DATA Modeled on the Diagonal of the Measurement AREa. The Phantom Contained 277 Cubes Randomly DistRibuted Each With the SAME MAGNETIC MOMENT

\begin{tabular}{|c|c|c|c|}
\hline Parameters & $\begin{array}{c}\text { Data at } \\
\{12,12\}\end{array}$ & $\begin{array}{c}\text { Data at } \\
\{20,20\}\end{array}$ & $\begin{array}{c}\text { Data at } \\
\{36,36\}\end{array}$ \\
\hline$A(-)$ & 0.0899 & 0.0692 & 0.1395 \\
\hline$b(\mathrm{~cm})$ & 4.2398 & 6.3591 & -2.0986 \\
\hline$c(\mathrm{~cm})$ & 16.1963 & 0.2719 & 15.2829 \\
\hline$D(T / m)$ & $-2.3678 \times 10^{-12}$ & $-2.3370 \times 10^{-12}$ & $-4.3988 \times 10^{-12}$ \\
\hline
\end{tabular}

a dipolar character and the fitting at the nodes provides the same center and constant $A$. It suggests that the last gradient in this configuration is almost redundant. However, this redundancy is welcome in the calculation, where it helps to reduce the error of the least square fit to the equation.

\section{DISCUSSION AND CONCLUSION}

We have built an experimental gradiometer with six sensors placed coaxially on a tight pair of copper wires. A novel multiple gradient measurement method allows us to determine parameters of small compact sources even out of a single measurement. Determination of bigger sources and complex deposits requires measurement in multiple nodes. We have shown that the new method brings more information for the neural network inversion process [5], [10], [11] in comparison to the previously used single gradient array. The iterative optimization process, applied in reasonable resolution, may now have a unique solution, because the number of degrees of freedom was significantly reduced.

\section{REFERENCES}

[1] D. Cohen, "Measurements of the magnetic fields by the human heart, brain and lungs," IEEE Trans. Magn., vol. 11, pp. 694-700, 1975.

[2] P. Ripka and P. Navratil, "Fluxgate sensor for magnetopneumometry," Sens. Actuators A, vol. 60, pp. 76-79, 1997. 
[3] P. Ripka, P. Kašpar, A. Tipek, and K. Švábová, "Magnetic lung diagnostics using fluxgate," in Proc. Imeko World Congress, Vienna, 2000, vol. VII, pp. 101-104.

[4] A. Tipek, "Vector magnetometers and their calibration," Ph.D. dissertation, CTU FEE, Prague, Czech Republic, 2002.

[5] J. Tomek, A. Platil, P. Ripka, and P. Kašpar, "Application of fluxgate gradiometer in magnetopneumography," Sens. Actuators, vol. 132, no. 1, pp. 214-217, 2006.

[6] F. Boháková and I. Šimáček, "SQUID magnetopneumography used to estimate the ferromagnetic particle content in the human lungs," $J M M M$, vol. 267, pp. 357-365, 2003.

[7] F. Boháková, I. Simáček, and P. Jurdák, "Basic quantification of magnetic particles in solid substance and human tissue by the SQUID magnetometer," Sens. Actuators A, vol. 129, pp. 150-153, 2006.

[8] G. Stroink, "Inverse problem solution in magnetisation studies," Phys. Med. Biol., vol. 32, no. 1, pp. 53-58, 1987.

[9] M. Forsman and P. Högstedt, "Comparison of multipole and mean value methods to quantify dust in human lungs: Simulating the MPG procedure," Med. Biol. Eng. Comput., vol. 36, pp. 452-460, 1998.

[10] J. Tomek, A. Platil, and P. Ripka, "Magnetopneumography - Advances in measurement procedure, modeling and inversion using artificial neural networks," in Proc. IJAEM, 2007, vol. 25, ISEM 2005, pp. 401-406, ISSN 1383-5416, Numbers 1-4, ISBN 978-1-58603-746-8.

[11] J. Tomek, A. Platil, and P. Ripka, "Application of neural networks inversion in Magnetopneumography," in Proc. IJAEM, 2007, vol. 26, OIPE 2006, pp. 285-290, ISSN 1383-5416, Number 3-4.

[12] A. Platil and J. Tomek, "Magnetic field inversion for magneto-pneumography," EMSA, Special Issue EMSA Sensor Letters, 2008, Caen, to be published.

[13] Y. Li and D. W. Oldenburg, "3-D inversion of magnetic data," Geophysics, vol. 61, pp. 394-408, 1996.

[14] M. Popov, "Data continuation for the explicit solution of an inverse biomagnetic problem," IEEE Trans. Magn., vol. 38, pp. 3620-3632, Nov. 2002.

[15] G. R. Barnes, P. L. Furlonga, K. D. Singha, and A. Hillebranda, "A verifiable solution to the MEG inverse problem," NeuroImage, vol. 31, no. 2, pp. 623-626, Jun. 2006.

[16] A. I. Braginski, J. A. Clarke, and J. Clarke, "The SQUID handbook," in Fundamentals and Technol.. New York: Wiley, 2004, vol. I, p. ISBN:3527604588.

[17] J. Vrba and S. E. Robinson, "Signal processing in magnetoencephalography," Methods, vol. 25, no. 2, pp. 249-271, Oct. 2001.

[18] J. Vrba and S. E. Robinson, "SQUID sensor array configurations for magnetoencephalography applications," Superconductor Sci. Technol., vol. 15, no. 9, pp. R51-R89(1), 2002.

[19] J. Vrba, S. E. Robinson, and J. McCubbin, "How many channels are needed for MEG?," in Neurology and Clinical Neurophysiology, 2004, vol. 99, doi:10.1016/j.ics.2007.01.061.

[20] J. Vrba and H. Wilsona, "Comparison of external noise cancellation in MEG," in Proc. Int. Congr. Series, Jun. 2007, vol. 1300, pp. 603-606, doi:10.1016/j.ics.2007.01.061E

[21] A. I. Braginski, J. A. Clarke, and J. Clarke, "The SQUID handbook," in Noise Supression. New York: Wiley, 2004, vol. I, ch. 7.7, p. ISBN: 3527604588

[22] Y. Adachi, J. Kawai, M. Miyamoto, G. Uehara, H. Ogata, and S. Kawabata, "Multichannel SQUID system for measurement of spinal cord evoked magnetic field for supine subjects," J. Physics: Conference Series, vol. 97, p. 6, 2008, doi:10.1088/1742-6596/97/1/012281.
[23] J. M. G. Merayo, J. R. Petersen, O. V. Nielsen, F. Primdahl, and P. Brauer, "A portable single axis magnetic gradiometer," Sens. Actuators A: Physical, vol. 93, no. 3, pp. 185-196, Oct. 2001.

[24] A. A. Garachtchenko, A. Matlashov, R. H. Kraus, Jr., and R. Cantor, "Baseline distance optimization for SQUID gradiometers," IEEE Trans. Appl. Superconduct., vol. 9, pp. 3676-3679, Jun. 1999.

[25] P. Volegov, A. Matlachov, J. Mosher, M. A. Espy, and R. H. Kraus, Jr., "Noise-free magnetoencephalography recordings of brain function," in Phys. Med. Biol., 2004, vol. 49, pp. 2117-2128, doi: 10.1088/00319155/49/10/020.

[26] J. Včelák, "Calibration of triaxial fluxgate gradiometer," J. Appl. Phys., vol. 99, no. 8, p. Doi:10.1063/1.2176262, 2006.

[27] P. Ripka, A. M. Lewis, P. Kaspar, and J. Vcelak, "DC gradiometers for bomb detection: Scalar versus vectorial," Sensor Lett., vol. 5, no. 1, pp. 271-275(5), Mar. 2007.

[28] R. Fitzpatrick, Classical electromagnetism: an intermediate level course, The Univ. Texas, Austin, TX, 2006. [Online]. Available: http://farside.ph.utexas.edu/teaching/em/lectures/node39.html

[29] P. Ramuhalli, L. Udpa, and S. S. Udpa, "Electromagnetic NDE signal inversion by function-approximation neural network," IEEE Trans. Magn., vol. 38, pp. 3633-3642, Nov. 2002.

Jiří Tomek received the B.Sc. degree from the Department of Measurement and the M.Sc. equivalent degree in biomedical engineering from the Faculty of Electrical Engineering, Czech Technical University, Prague, Czech Republic, in 2003 and 2005, respectively. He is currently working towards the Ph.D. degree in the Department of Measurement, Czech Technical University.

He is with the Medical Technique Service Department, General Teaching Hospital, Prague. He mainly deals with biomedical applications of magnetic measurements and inverse problems.

Antonin Platil graduated from the Czech Technical University, Faculty of Electrical Engineering, Prague, in 1997, and received the Ph.D. in 2002 from the Department of Measurement, Czech Technical University, Faculty of Electrical Engineering, Prague, Czech Republic.

Currently, he is a Researcher in the Department of Measurement and mainly deals with various aspects of magnetic measurements, sensors, and systems.

Pavel Ripka (M’00) received the Ing. degree (equivalent to M.Sc.) in 1984, the C.Sc. degree (equivalent to Ph.D.) in 1989, and the Prof. degree in 2001 from the Czech Technical University, Prague, Czech Republic.

He is the Head of the Department of Measurement, Faculty of Electrical Engineering, Czech Technical University. His main research interests are magnetic sensors and magnetometers. He is the author of more than 120 papers, 2 books, and 5 patents.

Prof. Ripka is a member of the Elektra Society, Czech Metrological Society, Czech National IMEKO Committee, and Eurosensors Steering Committee. He was the General Chairman of the Eurosensors 2002 Conference. 